\title{
Reduced Susceptibility to Fluoroquinolone in Salmonella typhi and Some Molecular Characteristics of Fluoroquinolone Resistance in Indigenous Isolates
}

\author{
Abu Sayeed M Mahmud, M Nuruddin Mahmud and M Abdul Hakim* \\ Department of Microbiology, University of Chittagong, Bangladesh.
}

(Received 16 August 2010; Accepted 9 December 2010)

\begin{abstract}
Typhoid fever and Salmonella typhi bacteremia among the patients were evaluated at Dhaka Shishu Hospital, Popular Diagnostic Center, Kumudiny Hospital and other sites throughout Bangladesh. Among the 943 Salmonella typhi isolates studied, $57.4 \%$ showed resistance to ampicillin (MIC > $256 \mathrm{mg} / \mathrm{ml}$ ), $58 \%$ to cotrimoxazole (MIC > $32 \mathrm{mg} /$ $\mathrm{ml}$ ) and $58.6 \%$ to chloramphenicol (MIC $>256 \mathrm{mg} / \mathrm{ml})$. Significant resistance $(\mathbf{9 7 . 8 \% )}$ ) was observed against first generation quinolone, nalidixic acid (MIC > $256 \mathrm{mg} / \mathrm{ml}$ ) whereas only 11 isolates $(1.1 \%)$ were resistant to second generation fluoroquinolone, ciprofloxacin. Among 943 isolates E-strip test of 411 isolates for ciprofloxacin showed that 53 isolates were very sensitive (MIC $<0.125 \mathrm{mg} / \mathrm{ml}$ ), 252 were sensitive (MIC between $0.125 \mathrm{mg} / \mathbf{m l}$ and $0.5 \mathrm{mg} /$ $\mathrm{ml}$ ), 95 were moderately sensitive (MIC between $0.5 \mathrm{mg} / \mathrm{ml}$ and $2.0 \mathrm{mg} / \mathrm{ml}$ ) and the rest were resistant (MIC > 2.0 $\mu \mathrm{g} / \mathrm{ml}$ ) or very highly resistant (MIC $512 \mathrm{mg} / \mathrm{ml}$ ). However, all isolates including ciprofloxacin resistant $S$. typhi were found sensitive to a third generation cephalosporin ceftazidime. All the isolates resistant to ciprofloxacin were found to be identical by API 20 E (Analytical Profile Index) score (4404552) and VNTR typing. Digestion of PCR product (195 bp gyrase A) of ciprofloxacin resistant strain with Hind I did not make any change, compared to the undigested product of the same strains in the electrophoresis banding pattern, indicating the presence of mutations at both the ser-83 and asp- 87 sites of the genome Gyr A. In contrast, the PCR product from nalidixic acid susceptible control strain cleaved at both sites, ser-83 and asp-87.
\end{abstract}

Key words: VNTR, Antibiotic resistance, Quinolone, Salmonella

\section{Introduction}

Salmonella enterica serovar Typhi (S. typhi) is the etiological agent of typhoid fever; a serious invasive bacterial disease of human with an annual global incidence of 21 million cases, with more than 700,000 deaths ${ }^{1}$. The indiscriminate use of fluoroquinolone has been accompanied by a frightening increase in the numbers of resistant Typhi. The emergence of MDR (multidrug resistant) Salmonella strains with resistance to fluoroquinolones and third-generation cephalosporins is a serious problem which results in severe limitation of the possibilities for effective treatment of human infections. Ciprofloxacin is considered as a first choice for treatment of typhoid fever in patients likely to have acquired infection in areas reporting multi drug resistant strains of Typhi ${ }^{2}$. With widespread use of the fluoroquinolones, selective pressure is exerted on patient populations, particularly those in intensive care and specialized care units in hospitals. This selective pressure results in the emergence of quinolone resistant bacteria ${ }^{3}$. The breakpoint value for reduced ciprofloxacin susceptibility was chosen as $>2.00 \mathrm{mg} /$ $\mathrm{mL}$ on the basis of earlier publications ${ }^{4}$ and our recent differential bactericidal concentration analyses, combined with the sequencing data of the quinolone resistance determining region (QRDR) of the gyrA gene ${ }^{5}$. Single or double mutation in the gyrase gene of Salmonella typhi is responsible for fluoroquinolone resistance. Quinolone resistance can also develop by mechanisms of reduced drug permeation and altered regulation of active efflux mechanisms ${ }^{6}$. Reduced susceptibility to fluoroquinolone in Salmonella serover Typhi and molecular aspect of fluoroquinolone resistance in Bangladeshi isolates was investigated in the present study.

\section{Materials and Methods}

Clinical sample

Blood culture of typhoid fever patients admitted to Dhaka Shishu hospital, Popular Diagnostic Center, Kumudiny Hospital and some other sites throughout Bangladesh.

\section{Cultivation of the bacteria}

S. typhi was isolated from cultures of blood only during the first 2 weeks of illness. The liquid medium used was trypticase soy supplemented peptone broth with an anticoagulant. The solid medium used was MacConkey agar (Himedia).

\section{Blood culture}

Five to ten milliliter of blood drawn from patients was poured into sterile cotton plugged bottles containing liquid medium, 
mixed well and allowed to flow over the agar on one of the narrow sides of bottle. The bottle was incubated in the upright position and the agar surface examined daily for colonies. Then the colonies were plated on MacConkey agar to obtain a pure culture ${ }^{7}$.

\section{Identification}

From the pure culture identification was carried out by typical biochemical tests and API 20E system (bioMérieux, Inc., Hazelwood, MO) $)^{8}$.

\section{Widal test}

The patients sera was tested by tube agglutination for the determination of titres of antibodies against $\mathrm{H}, \mathrm{O}$ and $\mathrm{Vi}$ antigen suspensions of the enteric fever bacteria likely to be encountered. Usually, antibodies to $\mathrm{O}$ antigen (IgM) appeared on days 6-8 and $\mathrm{H}$ antibodies (IgM and IgG) on days 10-12 after the onset of disease. The test was usually performed on an acute serum (at first contact with the patient) ${ }^{9}$.

\section{Susceptibility test}

MICs were determined by the standard agar plate dilution method according to the National Committee for Clinical Laboratory Standards (NCCLS) guidelines ${ }^{10}$. Mueller-Hinton II agar (BBL, Becton Dickinson, Cockeysville, MD, and USA) was used as the culture medium. The MIC breakpoints for resistance used were those recommended by the NCCLS ${ }^{10}$ except for fluoroquinolones. The resistance breakpoint for ciprofloxacin was chosen as $\geq 2.0 \mathrm{mg} / \mathrm{L}$ and for nalidixic acid as $\geq 32 \mathrm{mg} / \mathrm{L}$ on the basis of earlier reports ${ }^{2,11}$.

\section{Extraction of DNA}

From MacConkey agar two or three colonies were taken into an eppendorf tube and suspended into $500 \mathrm{~mL}$ of DNase free water. The bacterial suspension was placed on a heat stable floater and

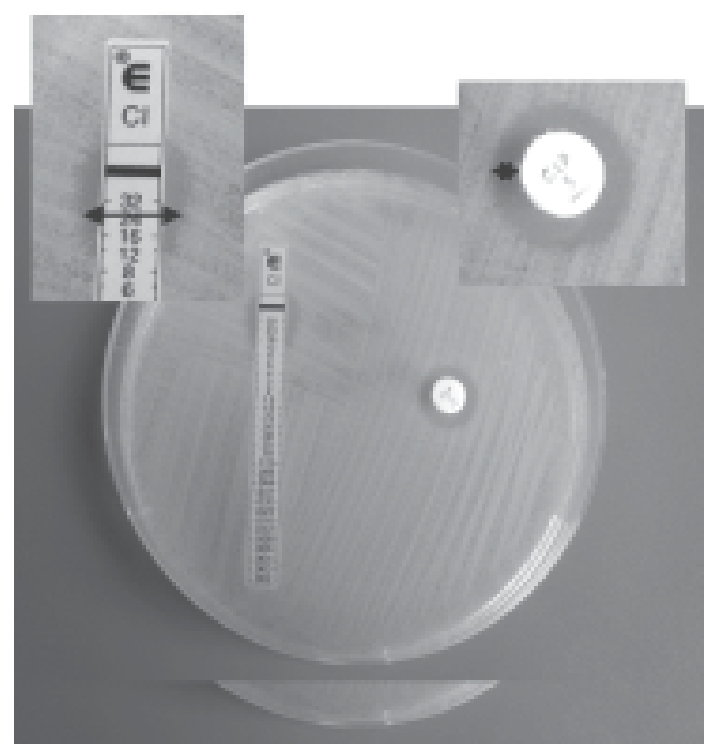

Fig 1: E-test for CIP resistant strain

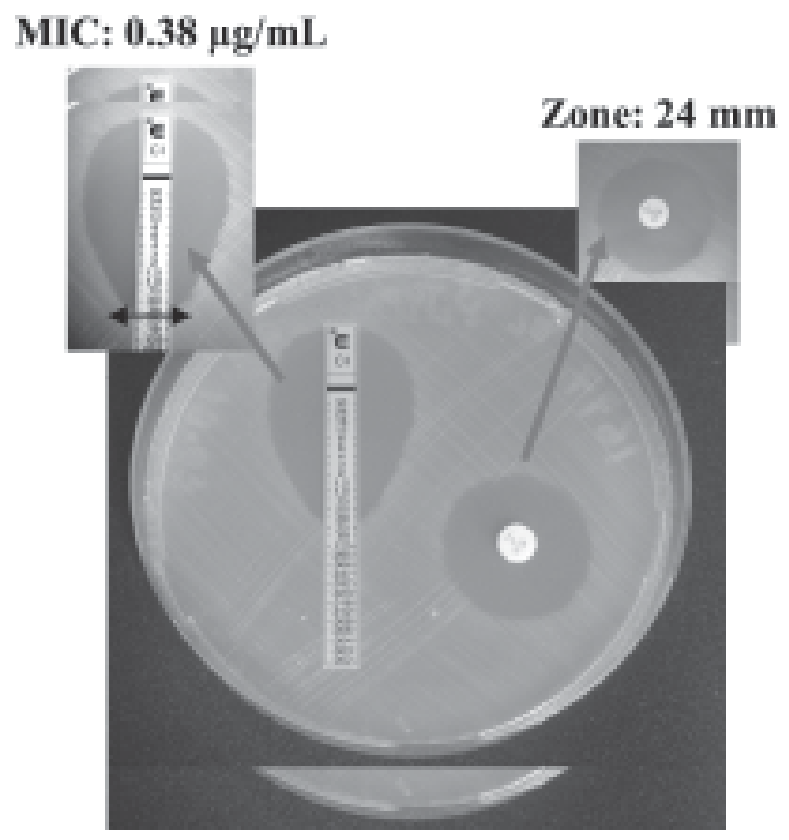

Fig 2: E-test of CIP sensitive strain

the whole apparatus was placed onto a boiling water bath for 10 minutes assuming that all the cells had swelled and burst releasing the genomic DNA ${ }^{12}$.

\section{VNTR for Molecular Typing}

Molecular typing was done by multiplex PCR assay using primers flanking three VNTR loci (TRI, TR2 \& TR3) ${ }^{12}$. After completion of the amplification of VNTR loci, the PCR products, along with a 100 bp DNA marker were subjected to electrophoresis on a 1.3 $\%$ agarose gel (Invitrogen, Carlsbad, CA, USA).

\section{Amplification of gyrase A gene}

The gyrF and gyrR primers were mixed with the PCR master mix along with template genomic DNA of both ciprofloxacin sensitive and ciprofloxacin resistant strains. Then the whole PCR mix was kept into the thermocycler for gyraseA gene amplification $^{12}$.

RFLP analysis: After completion of the amplification of gyrase A gene, the mixture was incubated with Hind I restriction endonuclease for 4 hours to overnight at $37^{\circ} \mathrm{C}$. After incubation, the digested mixture was mixed with $2 \mathrm{~mL}$ of gel loading buffer and subject to electrophoretic run through 3\% agarose gel in the electrophoresis unit ${ }^{12}$.

\section{Results}

Sensitivity pattern of Salmonella typhi isolates

Among the 943 isolates, $42.6 \%(\mathrm{n}=402)$ were sensitive to ampicillin (AMP) and 57.4\% ( $=541)$ were resistant; 42\% ( $n=$ 396) of the total isolates were sensitive to cotrimoxazole (CRO) and 57.4\% $(n=547)$ were resistant; and 41.4\% $(n=390)$ isolates were sensitive to chloramphenicol (CHL) and 58.6\% $(n=553)$ were resistant. Very low sensitivity was found with nalidixic acid 
which was only $2.2 \%(\mathrm{n}=21)$. On the other hand, Salmonella typhi isolates were found more sensitive to ciprofloxacin (CIP) and ceftazidime (CAZ). Only 1.1\% ( $\mathrm{n}=11)$ isolates showed resistance against ciprofloxacin whereas no resistant isolates was found against ceftazidime (figure: 3). E-strip test of 411 Salmonella typhi isolates for ciprofloxacin sensitivity showed that the MIC values varied among them. Fifty three strains (S1) had the MIC values nearer to the very sensitive $(<0.125 \mathrm{mg} /$ $\mathrm{ml}$ ), 252 isolates (S2) had a MIC between $0.125 \mathrm{mg} / \mathrm{ml}$ and 0.5 $\mathrm{mg} / \mathrm{ml}$, 95 isolates (S3) showed between $>0.5 \mathrm{mg} / \mathrm{ml}$ and 2.0 $\mathrm{mg} / \mathrm{ml}$ and the rest 11 strains (S4) had from $>2.0 \mathrm{mg} / \mathrm{ml}$ to very highly resistant MIC value for e.g. $512 \mathrm{mg} / \mathrm{ml}$. (figure: 4)

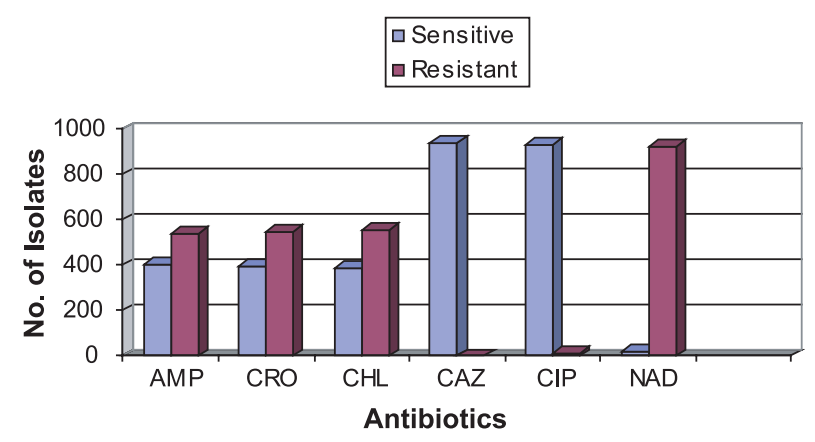

Fig 3: Response of Salmonella typhi to different antibiotics.

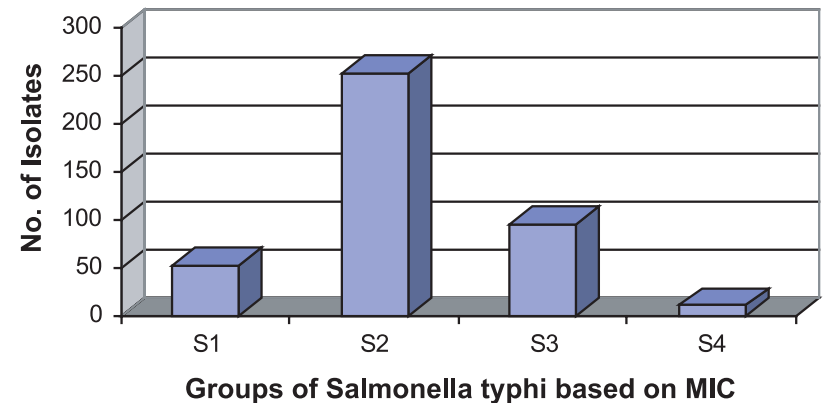

Fig 4: E-strip test of 411 Salmonella typhi isolates for ciprofloxacin sensitivity. S1(MIC $<0.125 \mathrm{mg} / \mathrm{ml}$ ), S2(MIC 0.125 $\mathrm{mg} / \mathrm{ml}$ to $0.5 \mathrm{mg} / \mathrm{ml}$ ), S3(MIC $0.5 \mathrm{mg} / \mathrm{ml}$ to $2.0 \mathrm{mg} / \mathrm{ml}$ ) S4(MIC $>2.0 \mathrm{mg} / \mathrm{m}$ to $512 \mathrm{mg} / \mathrm{ml}$ ).

Multiplex-PCR based variable number tandem repeat (VNTR) analysis

A total of 32 Salmonella isolates were analysed for VNTR banding profiles. Amplicons of different sizes were observed for isolates when they were subjected to multiplex PCR with primers for TR1, TR2, and TR3 (Figure 5). Visually each of these bands was observed similar in size to its counterpart obtained during amplification with individual primer pairs. The results showed that these 32 isolates could be segregated into 3 VNTR types. Most of the isolates had the same VNTR profile. Repetition of the multiplex PCR confirmed that the results were reproducible.
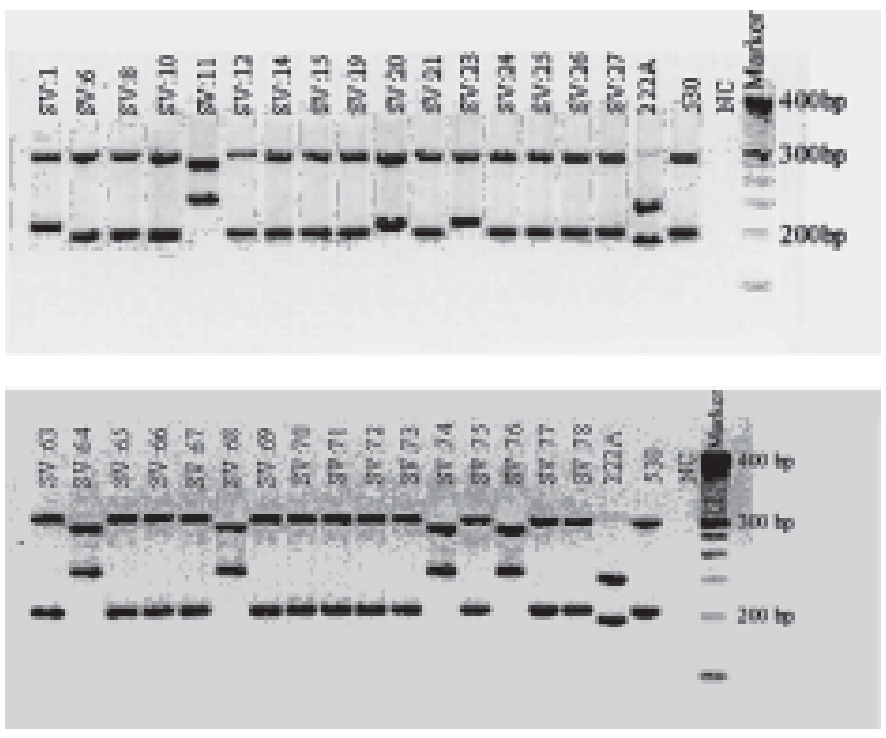

Fig 5: Banding pattern of Variable Number Tandem Repeats (VNTR) of Salmonella variants (SV) on agarose gel.

\section{RFLP Analysis for Determination of Mutation position}

On agarose gel, the gyrase Agenes of three ciprofloxacin resistant isolates were compared with that of reference ciprofloxacin resistant (positive control) and nalidixic acid sensitive (negative control) isolates (Figure: 6). The amplified product of ciprofloxacin resistant (high MIC) Salmonella typhi was digested by Hind I restriction enzyme. Positive control (+ve) contained 530 bp gyrase product which has two mutations at 87 and 83 codon position and is not digested after Hind I restriction enzyme treatment. Therefore, the $195 \mathrm{bp}$ of PCR product was visualized on agarose gel. Negative control (-ve) used was 243 bp (RE) product which was nalidixic acid sensitive with no mutation at 87 and 83 codon position that was digested after Hind restriction enzyme treatment and revealed 137 bp PCR product on agarose gel.

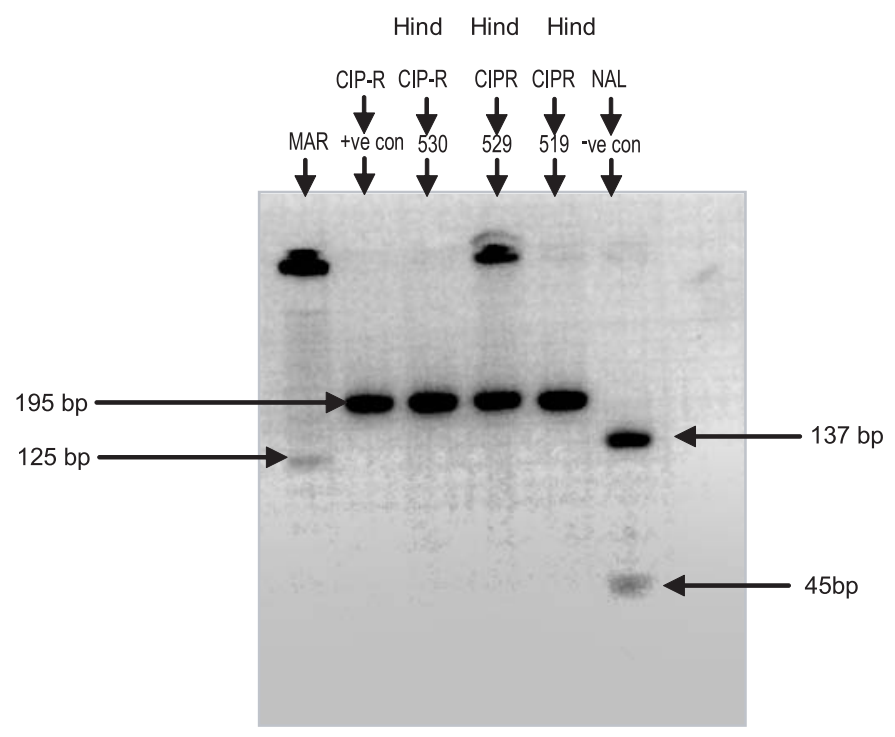

Fig 6: Band Patterns of Gyrase A gene (Digested by Hind l) on agarose gel. MAR indicates marker of known molecular weight. 


\section{Discussion}

Typhoid fever is endemic in Bangladesh. However, like many developing countries a reliable system to identify and to report this disease does not exist here. The present study showed a dramatic increase in the annual proportion of reduced fluoroquinolone susceptibility of Salmonella typhi in Bangladesh which changed from $3.9 \%$ to $23.5 \%$. The proportion of reduced susceptibility was similar to the isolates reported from Malaysia, Vietnam and India ${ }^{12-14}$. The common nature of quinolone resistance in South-East Asia has created tourist's risk of acquiring quinolone resistant salmonellosis. The emergence of antimicrobial resistance in any part of the world may have a global bearing and thus deserves universal attention. In Bangladesh, the possibility of clonal spread, a potential mechanism accounting for the high level of reduced fluoroquinolone susceptibility, as a major contributing factor was excluded in this study. In addition, some of the quinolone-resistant isolates contained different antimicrobial resistance patterns along with their different VNTR patterns. Based on these data, it can be concluded that the reduced fluoroquinolone susceptibility of Salmonella typhi in Bangladesh primarily involves mutations in chromosomal genes. This concept was consistent with the VNTR experiment. All 3 Salmonella isolates with reduced fluoroquinolone susceptibility so far analyzed by RFLP in our laboratory have shown point mutation leading to amino acid change in their QRDR of the gyrase A gene. The significantly more common multi-drug resistance observed here among the quinolone resistant salmonellae compared with the susceptible population (47.4\% vs. $11.5 \%)$ is also of concern. This finding suggests that the use of fluoroquinolones may select for multi-drug resistance among salmonellae and provokes a question of whether the same could happen among other bacterial species. In conclusion, it can be stated that reduced susceptibility of salmonellae to the fluoroquinolone group was significantly associated with multidrug resistance. Moreover, all quinolone resistant Salmonella isolates had undergone a point mutation in the QRDR of the gyrA gene. In contrast to previous report by Lee et al. on quinolone resistance in a specific clone or in a few Salmonella serotypes, the reduced fluoroquinolone susceptibility of our isolates was nonclonal ${ }^{15}$. The data provided in this report indicates evidence of the rapid spread of multidrug resistant pathogens from one continent to another.

\section{References}

1. Thong KL, Cheong YM, Puthucheary S, Koh CL and Pang T. 1994. Epidemiologic analysis of sporadic Salmonella typhi isolates and those from outbreaks by pulsed-field gel electrophoresis. J Clin Microbiol. 32:1135-1141.

2. Threlfall EJ, Ward LR and Rowe B. 1997. Increasing incidence of resistance to trimethoprim and ciprofloxacin in epidemic Salmonella typhimurium DT104 in England and Wales. Euro Surveillance 2: 81-4.

3. Pena C, Albareda JM, Pallares R, Pujol M, Tubau F and Arlza J. 1995 Relationship between quinolone use and emergence of ciprofloxacin resistant Escherichia coli in bloodstream infections. Antimicrob Agents Chemother. 39: 520-524.

4. Piddock LJ, Jin YF, Webber MA and Everett MJ. 2002. Novel ciprofloxacin resistant, nalidixic acid susceptible mutant of Staphylococcus aureus. Antimicrob Agents Chemother. 46: 2276-2278

5. Kustner FIGV. 1979. Trends in four major communicable diseases. SAfrica Med J. 55: 460-73.

6. Kohler T and Pechere JC. 1998. Bacterial resistance to quiriolones: mechanisms and clinical implications. $2^{\text {nd }}$ edn. pp 117-142. Academic Press, San Diego.

7. Garcia-Rodriguez JA, Garcia Sanchez JE, Munoz Bellido JL and Canut Blasco A. 1988. Comparison of a biphasic system and nonradiometric system for blood culture. Eur J Clin Microbiol Infect Dis. 7: 666-668.

8. Marymont JH, Marymont JH Jr. and Gavan T J. 1978. Performance of Enterobacteriaceae identification systems: an analysis of College of American Pathologists survey data. Am J Clin Pathol. 70: 539-547.

9. Dubey RC and Maheshwari DK. 2004. Practical Microbiology, $1^{\text {st }}$ edn, pp- 272. S. Chand \& Company Ltd, New Delhi-110 055

10. National Committee for Clinical Laboratory Standards.1997. Methods for Dilution Antimicrobial Susceptibility Tests for Bacteria that Grow Aerobically, $4^{\text {th }}$ Edn: Approved Standard M7-A4. NCCLS, Wayne, PA.

11. Frost JA, Kelleher A and Rowe B. 1996. Increasing ciprofloxacin resistance in salmonellas in England and Wales 1991- 1994. J Antimicrob Chemother. 37: 85-91.

12. Liu Y, Lee MA, Ooi EE, Mavis Y, Tan AL and Quek HH. 2003. Molecular Typing of Salmonella enterica Serovar Typhi Isolates from Various Countries in Asia by a Multiplex PCR Assay on Variable-Number Tandem Repeats. J Clin Microbiol. 41(9): 4388-4394.

13. Jesudason MV and Jacob TJ. 1990. Multiresistant Salmonella typhi in India. Lancet. 336: 252.

14. Threlfall EJ, Ward LR, Rowe B, Ranghupathi S, Chandrasekaran V, Vandepitte J and Lemmens P. 1992. Widespread occurrence of multiple drug resistant Salmonella typhi in India. Eur J Clin Microbiol Infect Dis. 11: 990-993.

15. Lee K, Lee M, Lim J, Jung J, Park Y and Lee Y. 2008. Contamination of chicken meat with Salmonella enterica Serovar Haardt with nalidixic acid resistance and reduced fluoroquinolone susceptibility. J Microbiol Biotechnol. 18(11): 1853-7. 